\title{
Racial/ethnicity disparities in invasive breast cancer among younger and older women: An analysis using multiple measures of population health
}

\author{
Mei-Chuan Hung, PhD, MS, RN, Donatus U. Ekwueme, PhD, MS ${ }^{\star}$, Sun Hee Rim, PhD, MPH, \\ and Arica White, PhD, MPH \\ Division of Cancer Prevention and Control, US Centers for Disease Control and Prevention, \\ Atlanta, Georgia
}

\section{Abstract}

Introduction-Few studies have examined age and racial/ethnic disparities in invasive breast cancer among younger (age 15-44 years) vs. older (age 45-64 years) women. This study estimates disparities in breast cancer among younger compared with older women by race/ethnicity using five measures of population health: life expectancy (LE), expected years of life lost (EYLL), cumulative incidence rate (CIR), and incidence and mortality rate ratios (IRR and MRR).

Methods-Using Surveillance, Epidemiology, and End Results data, LE and EYLL were estimated from a cohort of 15-44 and 45-64 years, non-Hispanic black (NHB), non-Hispanic white (NHW), and Hispanic women diagnosed with breast cancer, 2000-2013. Survival function was obtained from the study years and then extrapolated to lifetime using the Monte Carlo method. The CIR, IRR and MRR were calculated using 2009-2013 breast cancer incidence and mortality rates from the Centers for Disease Control and Prevention's National Program of Cancer Registries.

Results-The estimated LE ranged from 32.12 to 7.42 years for localized to distant stages among younger NHB women compared to 33.05 to 9.95 years for younger NHW women. The estimated EYLL was 12.78 years for younger women, and 4.99 for older women. By race/ ethnicity, it was 15.53 years for NHB, 14.23 years for Hispanic and 11.87 years for NHW ( $P$ $<0.00025)$. The CIR for age-group $15-44$ years $\left(\mathrm{CIR}_{15-44}\right.$ indicated a 1 in 86 probability for NHB compared to a 1 in 87 probability for NHW being diagnosed with breast cancer by age 45 . The

*Corresponding author at: Division of Cancer Prevention and Control, Centers for Disease Control and Prevention, 4770, Buford Highway, MS F-76, Atlanta, GA, 30341, Georgia. donatus.ekwueme@cdc.hhs.gov (D.U. Ekwueme).

Authorship contribution statement

In this revised manuscript, all the authors have made 1) substantial contributions to the conception and design or data analysis and interpretation; 2) contributed in addressing reviewers' comments; 3) drafting and revisions of the revised manuscript; and 4) approval of the final revised version submitted for consideration to be published in this journal.

Conflict of interest statement

None.

Financial disclosure

None.

Disclaimer

The findings and conclusions in this paper are those of authors and do not necessarily represent the official position of the Centers for Disease Control and Prevention. 
estimated age-adjusted incidence rate for NHB-to-NHW women was IRR $=1.10(95 \%, \mathrm{CI}=1.08$ $1.11)$ and the corresponding mortality rate was $\mathrm{MRR}=2.02(95 \%, \mathrm{CI}=1.94-2.11)$.

Conclusions-The breast cancer disparities between younger NHB compared to NHW women highlight the need for expanded efforts to address these disparities through primary prevention and to improve access to quality healthcare to minority women with breast cancer.

\section{Keywords}

Breast cancer; Racial health disparity; Incidence and mortality; Survival; Life expectancy; and expected years of life lost

\section{Introduction}

Breast cancer is the most common malignant tumor among U.S. women, accounting for $30 \%$ of incident cancers [1]. Despite the benefits of advances in treatment and early detection that have contributed to the decline in breast cancer deaths [2], not all age and racial/ethnic groups have benefited equally and disparities in incidence and mortality still exist [3]. Further, several studies have examined age disparities in breast cancer outcomes [4-9]. These studies have reported substantially lower health-related quality of life resulting from breast cancer treatment in younger (i.e., aged 15-44 years) compared with older (i.e., aged 45-64 years) women. Similarly, studies have reported racial/ethnic disparities in breast cancer outcomes [10-13]. These studies found that black women are more likely than white women to have late-stage breast cancer at diagnosis and have less favorable outcomes, including higher mortality rates [10,11]. In addition, other studies have also found that both younger and older black women have higher mortality rates than any other racial/ethnic group $[12,13]$.

In recent years, there has been public health initiative in breast cancer among younger women. More specifically, the Education and Awareness Requires Learning Young (EARLY) Act, Section 10413 of the Affordable Care Act [Public Law 111-148]) [14] has renewed interest in how the risk of breast cancer impacts younger women. Currently, no studies have examined age (younger versus (vs) older) and racial/ethnic disparities (that is, non-Hispanic black (NHB), non-Hispanic white (NHW), and Hispanic women) using multiple measures of population health, which includes life expectancy (LE), expected years of life lost (EYLL), cumulative incidence rates for women ages 15-44 and 45-64 years (CIR $15-44,45-64)$, and age-adjusted incidence and mortality rate ratios (IRR and MRR). However, the impact of breast cancer disparities have been quantified using other population-based measures developed to evaluate cancer progress in national health objectives [15-17].

In this paper, we utilized multiple measures of population health (i.e., LE, EYLL, CIR, IRR, and MRR), to determine 1) whether breast cancer burden was greater for younger vs older women; 2) to estimate the magnitude of racial/ethnic disparities in invasive breast cancer among younger NHB vs NHW women, and younger Hispanic vs NHW women; and 3) to examine whether racial/ethnic disparities in incidence and mortality of breast cancer among younger women are worse in breast cancer than in all other female cancers. Each of these 
measures contributes differently in providing information to patients, clinicians, and health policy-makers on the burden of breast cancer disparities in age-and racial/ethnic-groups. We hope that the use of these measures may provide useful data for outcome assessment and resource allocation of health services and development of prevention strategies for age- and racial/ethnic-groups that are disproportionately more likely to develop breast cancer in their lifetime.

\section{Methods}

\subsection{Data sources and study population}

Data from the National Cancer Institute (NCI)'s 2000-2013 Surveillance, Epidemiology, and End Results (SEER-18) registries [18], which covers 28\% of the US population, were used to estimate LE and EYLL. The SEER-18 registries were used because it contains survival information needed to estimate these measures [18]. A cohort of 88,406 women aged 15-44 and 345,025 women aged 45-64 with invasive breast cancer were identified from SEER. In addition, the combined dataset from the Centers for Disease Control and Prevention (CDC)'s National Program of Cancer Registries (NPCR) and the NCI's SEER registries [1], which covers the entire U.S. population, were used to estimate CIRs, incidence, and mortality rates for a separate cohort of 113,328 women aged 15-44 and 512,511 women aged 45-64 diagnosed (2009-2013) with invasive breast cancer. These population-based cancer registries collect detailed information on patient's sex, race/ ethnicity, year of diagnosis, age of diagnosis, cancer site, stage, survival months, and vital status [18]. In both datasets, invasive breast cancer was defined by the standards in the International Classification of Diseases for Oncology, 3rd Edition [19]. To minimize confounding of survival times for the primary cancer, patients with a prior breast cancer diagnosis and those diagnosed based on death certificate or autopsy only were excluded $(<1 \%)$. Death was defined as all-cause mortality; all breast cancer patients were followed until death or through the end of follow-up (December 31, 2013).

\subsection{Measures of population health}

The LE and EYLL incorporate data on the US life tables to estimate the burden of breast cancer in the population [20]. The estimation of LE from the date of diagnosis until death was used to generate lifetime survival in different racial/ethnic groups (NHB, NHW, and Hispanic) that may be relevant to clinicians in treating breast cancer patients. In estimating LE, we did not include racial/ethnic groups of Asian/Pacific Islander and American Indian/ Alaska Native women because of a lack of life tables for these populations.

On the other hand, the estimation of EYLL, which is a measure of the overall burden of disease was used to determine the lifetime health impact of breast cancer in age-and racial/ ethnic-groups. These two measures were used to examine the extent to which a younger NHB vs NHW, and younger Hispanic vs NHW woman's life span was likely to be reduced by breast cancer after diagnosis. The third measure $\left(\mathrm{CIR}_{15-44,45-64}\right)$ estimated age, and racial/ethnic disparities in the cumulative risk of being diagnosed with breast cancer over a time period and age interval [21] in the population. The last two measures, IRR and MRR, were used to further explore whether racial/ethnic disparity in incidence and mortality of 
breast cancer among younger women are worse in breast cancer than in all other female cancers [22].

\subsection{Calculation of life expectancy (LE)}

The Kaplan-Meier method [23] was used to estimate survival function for each cohort of breast cancer patients with 14 years of follow-up. Using a semi-parametric method [24], the estimated survival functions were further extrapolated up to 720 months (60 years) to derive LE after breast cancer diagnosis. The method uses relevant external information to aid the survival extrapolation. Its technical details have been described elsewhere [25]. Briefly, for a breast cancer cohort, the US life tables [26] were used to generate survival times for an age-, sex- and race/ethnicity-matched reference population using a Monte Carlo method.

Thesurvival times are then used for estimating survival function of the reference population. Second, with the assumption of excess constant hazard, logit transformation of the survival ratios between the cohort and the reference population will approach a straight line [27]. Hence, a linear regression was fitted to the logit transformed survival ratios during some time period near the end of follow-up. Finally, the estimated regression line and the survival function of the reference population beyond the follow-up limit were used to extrapolate the lifetime survival function of the cohort. The standard errors of the estimated LE were obtained using a bootstrap method.

\subsection{Calculation of expected years of life lost (EYLL) per breast cancer patient}

EYLL was defined as the reduced LE due to breast cancer in younger vs older women, younger NHB vs NHW women and younger Hispanic vs NHW women from that of the agesex- and race/ethnicity-matched reference population. It was calculated by taking the difference in the areas under the long-term survival curves between the breast cancer cohort and the reference population as shown in Fig. 1a, b, and c.

\subsection{Calculation of cumulative incidence rates $\left(\mathrm{CIR}_{15-44,45-64)}\right.$}

The $\mathrm{CIR}_{15-44,45-64}$ was calculated using the most recent five years of data (2009-2013) on breast cancer incidence rates among younger vs older women, younger NHB vs NHW women, and younger Hispanic vs NHW women from the NPCR/SEER registries [1]. The calculation of $\mathrm{CIR}_{15-44,45-64}$ was as follows: $\mathrm{CIR}_{15-44,45-64}=1-\exp \left[-S_{i}\left(\mathrm{IR}_{\mathrm{i}}\right) *\left(\Delta \mathrm{t}_{\mathrm{i}}\right)\right]$; where $\mathrm{IR}_{i}$ represents the age-specific incidence rate and $\Delta t_{i}$ indicates the range of each age stratum.

\subsection{Calculation of breast cancer incidence and mortality rate ratios (IRRs and MRRs)}

Using the age-adjusted breast cancer incidence and mortality rates obtained from the 20092013 NPCR-SEER registries [1], IRRs and MRRs for younger-to-older, younger NHB-toNHW and younger Hispanic-to-NHW women were calculated. We also calculated IRRs and MRRs for all other female cancer sites.

\subsection{Statistical analysis}

The methods described above for estimating LE and EYLL were conducted using the Integration of Survival with Quality of Life (iSQoL) statistical software [28]. Differences in 
stage distribution and EYLL of breast cancer patients by age-groups and race/ethnicity were tested with chi-square test and one-sided z-tests, with $P<0.05$ as the level of significance. Further, SEER*Stat software (version 8.3.2) [29] was used to extract age- and race/ethnicityspecific breast cancer incidence, mortality, and associated population data for the years 2009-2013. Rates, expressed per 100,000 population, were directly age-adjusted to the 2000 US standard population [30]. The age-adjusted IRRs and MRRs were also calculated using the age-adjusted rates for younger NHB vs NHW and younger Hispanic vs NHW women. We calculated $95 \%$ confidence intervals (CIs) for age-adjusted RRs based on methods described elsewhere [31].

\section{Results}

Table 1 presents the characteristics of invasive breast cancer by stage, age and race/ethnicity. Overall, the mean age at diagnosis for younger vs older women were 39.02 and 54.86 years, respectively. A significantly greater proportion of younger women $(48.13 \%)$ were diagnosed at late stages (i.e., regional and distant) compared with older women (38.66\%), $P<0.0001$. By racial/ethnic group, $54.49 \%$ of younger NHB women were diagnosed at late stage compared with $45.95 \%$ of NHW women, $(P<0.0001)$. Similarly, younger Hispanic women had a higher proportion of late stage diagnosis $(52.88 \%)$ compared with NHW women, $P$ $<0.0001$ (Table 1).

\subsection{Estimated LE and EYLL due to breast cancer}

For all women, the estimated LE was higher for younger women, with 33.36, 26.47, and 10.20 years for localized, regional, and distant stages, respectively, compared to estimates for older women, which were 26.26, 20.96, and 6.50 years for localized, regional, and distant stages, respectively, $P<0.00025$ (Table 2). The estimated LEs were shorter for younger NHB women $(32.12,22.01$, and 7.42 years for localized, regional and distant stages, respectively than estimates for NHW women, which were 33.05, 28.77, and 9.95 years for localized, regional, and distant stages, respectively. However, for younger Hispanic women the estimated LEs were longer than for NHW women in localized and distant stages (39.49 vs 33.05 and 12.17 vs 9.95 years), respectively.

Over a lifetime, younger women with breast cancer were estimated to have more life-years lost with an estimated EYLL of 12.78 years compared with 4.99 years for older women $P<0.00025$ (Fig. 1a). By racial/ethnic group, younger NHB women had more life-years lost with an estimated EYLL of 15.53 years compared with NHW women, $($ EYLL $=11.87$ years), $P<0.00025$ (Fig. 1b). Similarly, younger Hispanic women were more likely than NHW women to have greater life-years lost $(\mathrm{EYLL}=14.23$ vs. 11.87 year) (Fig. 1c).

By stage at diagnosis, the estimated life-years lost for all younger women were EYLL of $8.83,16.12$, and 33.15 years for localized, regional and distant stages compared with estimates for older women of 1.83, 7.76, and 21.51 years for localized, regional and distant stages, $P<0.00025$ (Table 2). Younger NHB women's EYLL was 7.89 and 18.42 for localized and regional stages compared with estimates for NHW women, EYLL were 9.30 and 13.95 years for localized and regional stages $(P<0.00025)$. The estimated EYLL was greater for younger Hispanic women than NHW women in regional and distant stages (20.36 
and 33.96 years). These estimates indicate that in all stages at diagnosis, younger women have more significant years of life lost than older women. Similarly, younger NHB women experience significantly more years of life lost at regional stage; however at localized stage, younger NHW women have slightly higher years of life lost.

\subsection{Estimated $\mathrm{CIR}_{15-44}, 45-64$ for breast cancer}

Overall, the CIR $15-44,45-64$ for younger women during the time period 2009-2013 was estimated to be 0.0109 vs 0.0479 for older women (Table 2). The results imply that among women aged 15-44 years, 1 in 92 will be diagnosed with an invasive breast cancer compared to 1 in 21 for women age 45-64 years. In other words, $1.09 \%$ of younger women would develop an invasive breast cancer by age 45 and $4.79 \%$ of older women would develop an invasive breast cancer by age 65 . By race/ethnicity, the $\mathrm{CIR}_{15-44}$ estimate for younger NHB women was 0.0116 , indicating that 1 in 86 will be diagnosed with an invasive breast cancer compared to 1 in 87 for younger NHW women $\left(\mathrm{CIR}_{15-44}=0.0115\right)$. For younger Hispanic women, the estimate was 0.0076 or 1 in 132 will be diagnosed with invasive breast cancer by age 45 .

\subsection{Incidence and mortality rate ratios}

Table 3 presents the age-adjusted IRR and MRR among younger NHB-to-NHW women, and younger Hispanic-to-NHW women by breast cancer and all other female cancer sites. Among younger women, the breast cancer incidence rate was 10\% higher for NHB than NHW (IRR $=1.10 ; 95 \% \mathrm{CI}=1.08-1.11$ ) (Table 3). On the other hand, the incidence rate for all other female cancer sites was significantly lower in NHB compared with NHW women (IRR $=0.70 ; 95 \% \mathrm{CI}=0.69-0.70)$. In general, the racial/ethnic disparity in incidence rate was worse in breast cancer than in all other female cancers. In contrast, the estimated breast cancer incidence rate and the rate for all other female cancers were lower in Hispanic than NHW women $(\operatorname{IRR}=0.73 ; 95 \% \mathrm{CI}=0.72-0.73$ and $\mathrm{IRR}=0.73 ; 95 \% \mathrm{CI}=0.72-0.73$, respectively.

The estimated mortality rate for breast cancer was twice as high for younger NHB compared with NHW women (MRR $=2.02 ; 95 \% \mathrm{CI}=1.94-2.11)$. Similarly, the rate for all other female cancer sites was also higher for NHB compared with NHW women $(\mathrm{MRR}=1.17$; $95 \% \mathrm{CI}=1.16-1.19)$. The disparity in mortality rate was worse in breast cancer than in all other female cancers. On the contrary, younger Hispanic women were significantly less likely than NHW women to die of breast cancer (MRR $=0.78 ; 95 \% \mathrm{CI}=0.73-0.82)$. A similar result was also observed in all other female cancers (MRR $=0.80 ; 95 \% \mathrm{CI}=0.79$ $0.81)$.

\section{Discussion}

Using multiple measures of population health, there were significant age and racial/ethnic disparities among younger women across five measures of population health. Compared with older women, a significant proportion of younger women have more years of life lost and a greater occurrence of being diagnosed with breast cancer at late stages. However, younger women have a lower risk of developing breast cancer. Further, relative to younger 
NHW women, a greater proportion of younger NHB women were diagnosed at late stages (i.e., regional and distant), had lower life expectancy, greater years of life lost, a higher probability of being diagnosed with breast cancer at an early age and a greater burden of age-adjusted incidence and mortality rates. Similarly, younger Hispanic women had a high rate of being diagnosed with breast cancer at late-stages, have a high proportion of years of life lost, but have less probability of being diagnosed with breast cancer as well as less likely to die from it compared with NHW women.

The findings from the use of these measures are consistent with previous literature on the age disparity $[4-9,32]$ and is also consistent with previous work on the racial/ethnic disparity and confirms that NHB women experience a higher burden of disease from breast cancer at a younger age $[4,10-13,33,34]$. To our knowledge, there are no other studies that have quantified the burden of breast cancer in younger vs older women, younger NHB vs NHW women, and younger Hispanic vs NHW women using these multiple measures of health. Prior to this paper, most studies examined racial/ethnic disparities in breast cancer, but they did not make race/ethnic-specific comparisons between younger versus older women $[10,11,35]$.

Currently, there are no studies that have quantified the burden of breast cancer in younger women using EYLL. However, compared with findings from older women, younger women experience greater years of life lost with an additional 7.79-year decrease in life span $[24,36]$. Consistent with shorter life expectancy observed among younger NHB women, the average years of life lost was greater for NHB and Hispanic than for NHW women, with NHB and Hispanic women experiencing an additional 3.36-year and 2.36-years decrease in life span.

We are not aware of any previously published estimates of $\mathrm{CIR}_{15-44}$ on breast cancer in younger women, but postulate that these estimates may increase in the future given recent studies reporting the increasing number of new cancer cases and widening disparities [3741]. The cumulative probability of breast cancer occurrence for women 15-44 years showed that more NHB women and fewer Hispanic than NHW women were diagnosed with an invasive breast cancer by age 45 .

Estimates of IRR and MRR revealed significant disparities in incidence and mortality rates for breast cancer and in all other female cancer sites. These findings are consistent with previous reports [22,38-41] and a reminder that regardless of improvement in breast cancer survivorship, racial/ethnic disparities still persistent. The disparities in incidence may reflect the greater decline in incidence for white women than for black women. Among white women, this decline is partly due to decreasing use of hormone replacement therapy (HRT) [42]. Black women did not experience this level of benefit because their use of HRT was low [42]. Further, a recent study has found a convergence of incidence rates between black and white women [43]. As reported in this paper, incidence rates increased from 2009 to 2013 for NHB women, while the rates for NHW women decreased. Further, breast cancer deaths for NHB women are expected to increase, which likely reflects the growing, aging population [44]. Many factors contribute to large mortality gaps. For instance, compared with white women, black women are more likely to be diagnosed at later stage as reported in 
this paper and less likely to receive timely and high quality treatment at the time of diagnosis [35,45-51]. Further, compared with white women, black women are more likely to have tumor subtypes, especially the triple negative subtype, which have poorer prognosis [52-56]. Unlike younger black women, younger Hispanic women are less likely to be diagnosed and die from breast cancer than non-Hispanic White women. This finding is consistent with previous reports $(40,42,60)$. However, there is a lot of variation within Hispanic subgroups and additional work is needed [57].

As demonstrated in this study, age and racial/ethnic disparities in breast cancer still remain. However, there have been efforts to address age- and racial/ethnic group disparities [58]. For example, the $\mathrm{CDC}$ has recently launch a multimedia, multi-pronged national campaign to educate younger women on the risk factors for breast cancer, encourage them to learn their family history of breast cancer, inspire them to talk to their healthcare provider if they think they might be at higher risk, and encourage them to live healthy lifestyles and be aware of their own breast health [59]. Given that these interventions are still in the nascent stages, the findings reported in this paper could be useful to inform decisions to effectively target populations with disproportionate burden of breast cancer. Additionally, the data reported in this paper could be used as inputs to conduct economic studies to evaluate the costeffectiveness of interventions designed to reduce breast cancer in younger women.

According to the Institute of Medicine, no single measure can sufficiently capture the health of the nation [60]. Therefore, the use of multiple measures of population health to quantify the magnitude of breast cancer disparities provides a more meaningful representation of the burden of breast cancer. Findings reported in each of these measures may be helpful for program planners and decision makers to inform allocation of resources and effectively target subgroups of the population with a disproportionate burden of breast cancer disease. More efforts are needed to detect cancer at an early, treatable stage and to ensure timely access to high quality treatment to reduce this disproportionate burden.

This study has some limitations. First, this study only provides estimates of the average loss of LE by cancer stage and did not consider other factors, such as comorbidity and disability, therefore, reported LE may be overstated [61,62]. Second, calculated CIR $15-44$ may be slightly overestimated because the approach assumes that the current risk estimates remain stable during the follow-up period (2009-2013) and the population under study is not subject to competing risk of death from other causes [63]. Finally, given that Hispanics are more likely to have incomplete follow-up for censored cases than whites or blacks in SEER dataset, estimates for Hispanic ethnicity should be interpreted with caution [64]. However, we used the active follow-up (reported alive method) to calculate their survival interval; hence Hispanics' survival may not be overestimated.

In conclusion, there are substantial disparities in breast cancer between younger compared with older women, and younger NHB women compared with younger NHW women in the multiple measures of population health used in this study. This suggests that many younger women, especially NHB are disproportionately impacted by breast cancer compared to their counterparts. Expanded efforts are needed to understand how to better address these disparities through primary prevention and improve access to quality healthcare. 


\section{Acknowledgments}

The authors sincerely thank Professor Jung-Der Wang MD, ScD, of the National Cheng Kung University College of Medicine and Dr. Jing-Shiang Hwang, PhD, of the Institute of Statistical Science, Academia Sinica, Taiwan for their helpful substantive comments on earlier versions of this manuscript.

\section{References}

1. U.S. Cancer Statistics Working Group. United States Cancer Statistics: 1999-2013 Incidence and Mortality Web-based Report. Atlanta: U.S. Department of Health and Human Services, Centers for Disease Control and Prevention and National Cancer Institute; 2016. [Accessed 14.07.16]

2. Berry DA, Cronin KA, Plevritis SK, Fryback DG, Clarke L, Zelen M, et al. Cancer Intervention and Surveillance Modeling Network (CISNET) collaborators: effect of screening and adjuvant therapy on mortality from breast cancer. N Engl J Med. 2005; 353:1784-1792. [PubMed: 16251534]

3. Bao Y, Fox SA, Escarce JJ. Socioeconomic and racial/ethnic differences in the discussion of cancer screening: between- versus within- physician differences. Health Serv Res. 2007; 42(3):950-970. (Pt 1). [PubMed: 17489898]

4. Trogdon JG, Ekwueme DU, Chamiec-Case L. Guy GP: breast cancer in young women: health state utility impacts by race/ethnicity. Am J Prev Med. 2016; 50(2):262-269. [PubMed: 26775905]

5. Brown DS, Trogdon JG, Ekwueme DU, Chamiec-Case L, Guy GP, Tangka FK, et al. Health-state utility impact of breast cancer in U.S. women aged 18-44. Am J Prev Med. 2016; 50(2):255-261. [PubMed: 26775904]

6. Kornblith AB, Powell M, Regan MM, Bennett S, Krasner C, Moy B, et al. Long-term psychosocial adjustment of older vs younger survivors of breast and endometrial cancer. Psychooncology. 2007; 16(10):895-903. [PubMed: 17245695]

7. Pritchard KI. Adjuvant therapy of the very young woman. Breast. 2007; 16:S136-S146. doi:http:// dx.doi.org/10.1016/j.breast.2007.07.023. [PubMed: 17766115]

8. Baucom DH, Porter LS, Kirby JS, Gremore TM, Keefe FJ. Psychosocial issues confronting young women with breast cancer. Breast Dis. 2005; 23:103-113. [PubMed: 16823173]

9. Arora NK, Gustafson DH, Hawkins RP, McTavish F, Cella DF, Pingree S, et al. Impact of surgery and chemotherapy on the quality of life of younger women with breast carcinoma: a prospective study. Cancer. 2001; 92(5):1288-1298. [PubMed: 11571745]

10. Centers for Disease Control and Prevention. Vital signs: Racial disparities in breast cancer severity —United States, 2005-2009. Morbid Mortal Wkly Rep. 2012; 61:922-926.

11. Parise CA, Caggiano V. Disparities in race/ethnicity and socioeconomic status: risk of mortality of breast cancer patients in the California Cancer Registry, 2000-2010. BMC Cancer. 2013; 13:449. doi:http://dx.doi.org/10.1186/1471-2407-13-449. [PubMed: 24083624]

12. McCarthy AM, Yang J, Armstrong K. Increasing disparities in breast cancer mortality from 1979 to 2010 for US black women aged 20 to 49 years. Am J Public Health. 2015:e1-e3.

13. Ademuyiwa FO, Gao F, Hao L, Morgensztern D, Aft RL, Ma CX, et al. US breast cancer mortality trends in young women according to race. Cancer. May 1; 2015 121(9):1469-1476. [PubMed: 25483625]

14. [Accessed on 10.08.16] Breast Cancer Education and Awareness Requires Learning Young (EARLY) Act of 2009. (H.R. 1740, S. 994). Available at: https://www.govtrack.us/congress/ bills/111/hr1740/text

15. Harper S, Lynch J, Meersman SC, Breen N, Davis WW, Reichman ME. An overview of methods for monitoring social disparities in cancer with an example using trends in lung cancer incidence by area-socioeconomic position and race-ethnicity, 1992-2004. Am J Epidemiol. 2008; 167(8): 889-899. [PubMed: 18344513]

16. Harper S, Lynch J, Meersman SC, Breen N, Davis WW, Reichman MC. Trends in areasocioeconomic and race-ethnic disparities in breast cancer incidence, stage at diagnosis, screening, mortality and survival among women ages 50 years and over (1987-2005). Cancer Epidemiol Biomarkers Prev. 2009; 18(1):121-131. [PubMed: 19124489] 
17. Harper, S., Lynch, J. [Accessed 10.07.15] Methods for Measuring Cancer Disparities: Using Data Relevant to Healthy People 2010 Cancer-Related Objectives. Available at: www.seer.cancer.gov/ archive/publications/disparities/measuring_disparities.pdf\#page=66

18. Surveillance, Epidemiology, and End Results (SEER) Program. Research Data (1973-2013). National Cancer Institute, DCCPS, Surveillance Research Program, Surveillance Systems Branch; (www.seer.cancer.gov)released April 2016, based on the November 2015 submission

19. International Classification of Diseases for Oncology, Third Edition, First Revision. Geneva: World Health Organization; 2013. Available at: http://codes.iarc.fr/ [Accessed 10.12.15]

20. Murray, CJL., Mathers, CD., Salomon, JA., Lopez, AD. Health gaps: an overview and critical appraisal. In: Murray, CJL.Salomon, JA.Mathers, CD., Lopez, AD., editors. Summary Measures of Population Health; Concepts, Ethics, Measurement and Applications. World Health Organization; Geneva: 2002. p. 233-244.

21. Breslow, NE., Day, ME. Statistical Methods in Cancer Research Volume I: the Design and Analysis of Cohort Studies. International Agency for Research on Cancer; Lyon, France: 1987.

22. Menashe I, Anderson WF, Jatoi I, Rosenberg PS. Underlying causes of the black-white racial disparity in breast cancer mortality: a population-based analysis. J Natl Cancer Inst. 2009; 101(14):993-1000. [PubMed: 19584327]

23. ADG. Practical Statistics for Medical Research. 2. Chapman \& Hall; London: 2006.

24. Hwang JS, Wang JD. Monte Carlo estimation of extrapolation of quality-adjusted survival for follow-up studies. Stat Med. 1999; 18(13):1627-1640. [PubMed: 10407234]

25. Chu PC, Wang JD, Hwang JS, Chang YY. Estimation of life expectancy and the expected years of life lost in patients with major cancers: extrapolation of survival curves under high-censored rates. Value Health. 2008; 11(7):1102-1109. [PubMed: 18489497]

26. Centers for Disease Control and Prevention. [Accessed 10.12.15] United States Life Tables. Available from:www.cdc/nchs/products/life_table.htm

27. Fang CT, Chang YY, Hsu HM, Twu SJ, Chen KT, Lin CC, et al. Life expectancy of patients with newly-diagnosed HIV infection in the era of highly active antiretroviral therapy. QJM. 2007; 100(2):97-105. [PubMed: 17277317]

28. Hwang, JS. [Accessed 10.07.15] iSQoL: Integration of Survival with Quality of Life (free Software). 2015. Available at: www.stat.sinica.edu.tw/isqol/

29. Surveillance Research Program, National Cancer Institute. [Accessed 14.07.16] SEER*Stat Software, Latest Release: Version 8.3.2. 2016. Available at: http://seer.cancer.gov/seerstat/ Seer*Stat

30. Anderson RN, Rosenberg HM. Age standardization of death rates: implementation of theyear 2000 standard. Natl Vital Stat Rep. 1998; 47(3):1-16. (20).

31. Tiwari RC, Clegg LX, Zou Z. Efficient interval estimation for age-adjusted cancer rates. Stat Methods Med Res. 2006; 15(6):547-569. [PubMed: 17260923]

32. Anders CK, Johnson R, Litton J, Phillips M, Bleyer A. Breast cancer before age 40 years. Semin Oncol. 2009; 36(3):237-249. [PubMed: 19460581]

33. Baquet CR, Mishra SI, Commiskey P, Ellison GL, DeShields M. Breast cancer epidemiology in blacks and whites: disparities in incidence, mortality, survival rates and histology. JNMA. 2008; 100(5):480-488.

34. Ekwueme DU, Guy GP Jr, Rim SH, White A, Hall IJ, Fairley TL, et al. Health and economic impact of breast cancer mortality in young women, 1970-2008. Am J Prev Med. 2014; 46(1):7179. [PubMed: 24355674]

35. Shavers VL, Brown ML. Racial and ethnic disparities in the receipt of cancer treatment. J Natl Cancer Inst. 2002; 94(5):334-357. [PubMed: 11880473]

36. Liu PH, Wang JD, Keating NL. Expected years of life lost for six potentially preventable cancers in the United States. Prev Med. 2013; 56(5):309-313. [PubMed: 23428566]

37. Weir HK, Thompson TD, Soman A, Møller B, Leadbetter S. The past, present, and future of cancer incidence in the United States: 1975 through 2020. Cancer. 2015; 121(11):1827-1837. [PubMed: 25649671]

38. Whitman S, Ansell D, Orsi J, Francois T. The racial disparity in breast cancer mortality. J Community Health. 2011; 36:588-596. [PubMed: 21190070] 
39. Jatoi I, Anderson WF, Rao SR, Devesa SS. Breast cancer trends among black and white women in the United States. J Clin Oncol. 2005; 23(31):7836-7841. [PubMed: 16258086]

40. U.S. Mortality Files NCHS, CDC. [Accessed 10.07.15] Female breast cancer death rates by race and ethnicity, U.S., 1999-2012. Available at: http://www.cdc.gov/cancer/breast/statistics/race.htm

41. Hunt BR, Whitman S, Hurlbert MS. Increasing Black:White disparities in breast cancer mortality in the 50 largest cities in the United States. Cancer Epidemiol. 2014; 38(2):118-123. [PubMed: 24602836]

42. Krieger N, Chen JT, Waterman PD. Decline in US breast cancer rates after the Women's Health Initiative: socioeconomic and racial/ethnic differentials. Am J Public Health. 2010; 100(Suppl 1):S132-S139. [PubMed: 20147667]

43. DeSantis CE, Fedewa SA, Goding Sauer A, Kramer JL, Smith RA, Jemal A. breast cancer statistics, 2015: convergence of incidence rates between black and white women. CA Cancer J Clin. Jan; 2016 66(1):31-42. [PubMed: 26513636]

44. Weir HK, Thompson TD, Soman A, Møller B, Leadbetter S, White MC. Meeting the healthy people 2020 objectives to reduce cancer mortality. Prev Chronic Dis. 2015; 12:140482.

45. Balasubramanian BA, Demissie K, Crabtree BF, Strickland PA, Pawlish K, Rhoads GG. Black medicaid beneficiaries experience breast cancer treatment delays more frequently than Whites. Ethn Dis. 2012; 22(3):288-294. [PubMed: 22870571]

46. Albain KS, Unger JM, Crowley JJ, Coltman CA Jr, Hershman DL. Racial disparities in cancer survival among randomized clinical trials patients of the Southwest oncology group. J Natl Cancer Inst. 2009; 101(14):984-992. [PubMed: 19584328]

47. Lund MJ, Brawley OP, Ward KC, Young JL, Gabram SS, Eley JW. Parity and disparity in first course treatment of invasive breast cancer. Breast Cancer Res Treat. 2008; 109:545-557. [PubMed: 17659438]

48. George P, Chandwani S, Gabel M, Ambrosone CB, Rhoads G, Bandera EV, et al. Diagnosis and surgical delays in African American and white women with early-stage breast cancer. J Womens Health. 2015; 24(3):209-217.

49. Smith EC, Ziogas A, Anton-Culver H. Delay in surgical treatment and survival after breast cancer diagnosis in young women by race/ethnicity. JAMA Surgery. 2013; 148(6):516-523. [PubMed: 23615681]

50. Institute of Medicine (IOM). Unequal Treatment: Confronting Racial and Ethnic Disparities in Health Care. Washington, DC: The National Academies Press; 2002.

51. U.S. Department of Health and Human Services. National Center on Minority Health and Health Disparities. [Accessed 10.07.15] Social Determinants of Health Initiative. Available at: http:// www.nimhd.nih.gov/recovery/goSocialDeterm.asp

52. Clarke CA, Keegan TH, Yang J, Press DJ, Kurian AW, Patel AH, et al. Age-specific incidence of breast cancer subtypes: understanding the black-white crossover. J Natl Cancer Inst. 2012; 104(14):1094-1101. [PubMed: 22773826]

53. Amirikia KC, Mills P, Bush J, Newman LA. Higher population-based incidence rates of triplenegative breast cancer among young African-American women: implications for breast cancer screening recommendations. Cancer. 2011; 117(12):2747-2753. [PubMed: 21656753]

54. Carey LA, Perou CM, Livasy CA, Dressler LG, Cowan D, Conway K, et al. Race, breast cancer subtypes, and survival in the Carolina breast cancer study. JAMA. 2006; 295(21):2492-2502. [PubMed: 16757721]

55. Howlader N, Altekruse SF, Li CI, Chen VW, Clarke CA, Ries LA, et al. US incidence of breast cancer subtypes defined by joint hormone receptor and HER2 status. J Natl Cancer Inst. 2014; 106(5):dju055. [PubMed: 24777111]

56. Yu Q, Fan Y, Wu X. General multiple mediation analysis with an application to explore racial disparity in breast cancer survival. J Biomet Biostat. 2014; 5(2):189.

57. Stern MC, Fejerman L, Das R, Setiawan VW, Cruz-Correa MR, Perez-Stable EJ, et al. Variability in cancer risk and outcomes within US latinos by national origin and genetic ancestry. Curr Epidemiol Rep. 2016; 3:181-190. [PubMed: 27547694]

58. Centers for Disease Control and Prevention. [Accessed 10.07.15] Breast Cancer in Young Women. Available at: http://www.cdc.gov/cancer/breast/young_women/index.htm 
59. CDC. [Accessed 05.02.16] Bring Your Brave Campaign. www.cdc.gov/cancer/breast/ young_women/bringyourbrave/index.htm

60. Institute of Medicine. State of the USA Health Indicators: Letter Report. Washington (DC): National Academies Press; 2009.

61. Keegan TH, Kurian AW, Gali K, Tao L, Lichtensztajn DY, Hershman DL, et al. Racial/ethnic and socioeconomic differences in short-term breast cancer survival among women in an integrated health system. Am J Public Health. 2015; 105(5):938-946. [PubMed: 25790426]

62. Repetto L, Comandini D, Mammoliti S. Life expectancy, comorbidity and quality of life: the treatment equation in the older cancer patients. Crit Rev Oncol Hematol. 2001; 37(2):147-152. [PubMed: 11166588]

63. Schouten LJ, Straatman H, Kiemeney LA, Verbeck AL. Cancer incidence: life table risk versus cumulative risk. J Epidemiol Comm Health. 1994; 48:596-600.

64. Pinheiro PS, Morris CR, Liu L, Bungum TJ, Altekruse SF. The impact of follow-up type and missed deaths on population-based cancer survival studies for Hispanics and Asians. J Natl Cancer Inst Monogr. 2014; 49:210-217. 

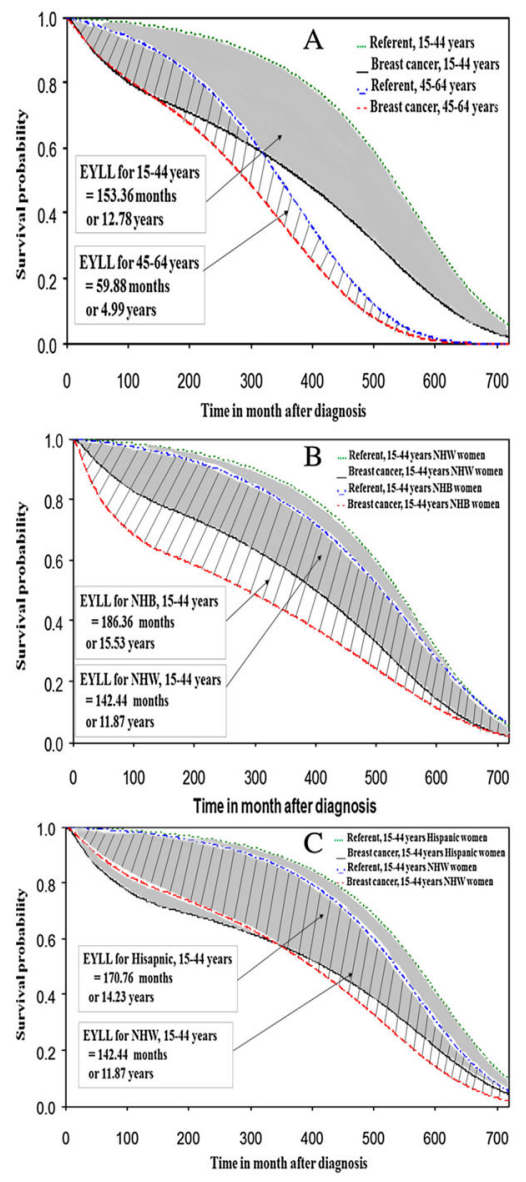

Fig. 1.

(a) Estimated expected years of life lost (EYLL) from breast cancer among younger women aged 15-44 years compared with older women aged 45-64 years, SEER registries, 20002013. ${ }^{1}$ (b) Estimated expected years of life lost (EYLL) from breast cancer among younger non-Hispanic black (NHB) women compared with younger non-Hispanic white (NHW) women, SEER registries, 2000-2013. ${ }^{2}$ (c) Estimated expected years of life lost (EYLL) from breast cancer among younger Hispanic women compared with younger non-Hispanic white (NHW) women, SEER registries, 2000-2013. ${ }^{3}$

${ }^{1}$ The estimated EYLL was 7.79 years (= 12.78-4.99 years) was significantly different between younger and older women with $P<0.00025$

${ }^{2}$ The estimated EYLL was 3.66 years $(=15.53-11.87$ years $)$ was significantly different between younger NHB women vs. younger NHW women with $P<0.00025$.

${ }^{3}$ The estimated EYLL was 2.36 years $(=14.23-11.87$ years) was significantly different between younger Hispanic women vs. younger NHW women with $P<0.00025$ 


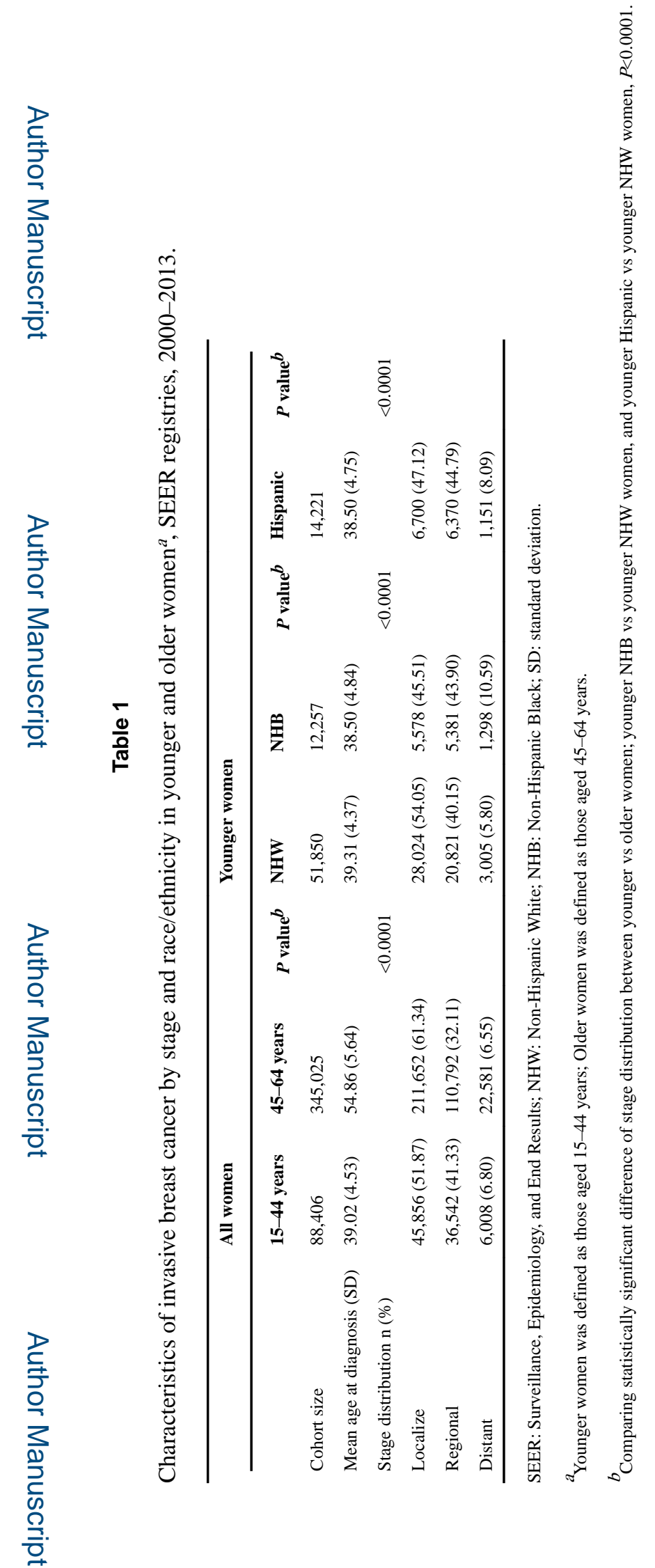

Cancer Epidemiol. Author manuscript; available in PMC 2018 March 21. 


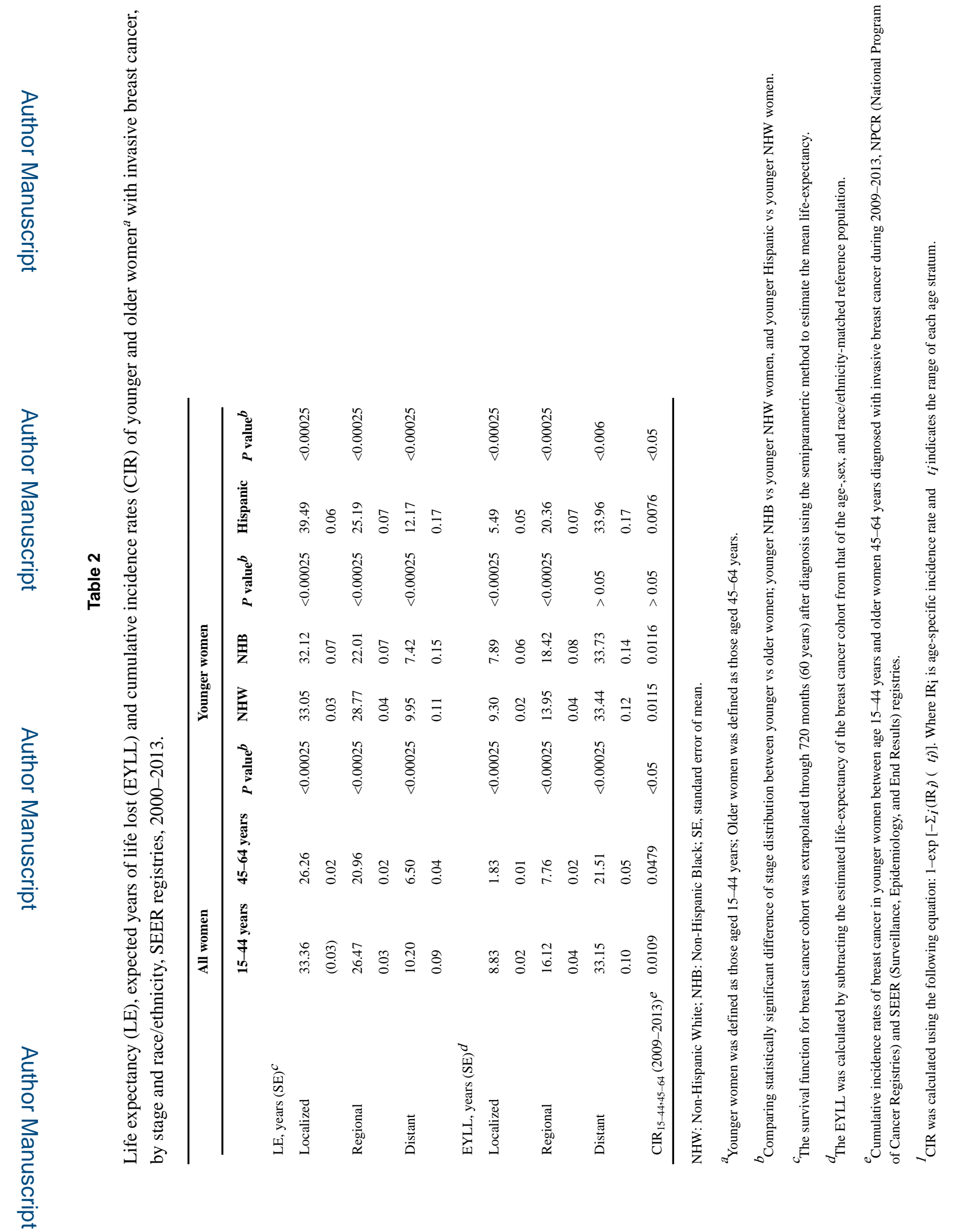

Cancer Epidemiol. Author manuscript; available in PMC 2018 March 21. 


\section{Table 3}

Estimated age-adjusted incidence and mortality rate ratios in breast and all female cancer sites among younger women ${ }^{a}$ by race/ethnicity, SEER and NPCR, 2009-2013.

\begin{tabular}{lllll}
\hline \multicolumn{3}{l}{ Incidence rate ratios } & \multicolumn{3}{l}{ Mortality rate ratio } \\
\hline & \multicolumn{2}{l}{ Race/ethnicity by younger women } & \multicolumn{2}{l}{ Race/ethnicity by younger women } \\
& $15-44$ years NHB: NHW & $15-44$ years Hispanic: & $15-44$ years NHB: NHW & $15-44$ years Hispanic: \\
& $\mathrm{RR}^{b}$ & NHW RR & $\mathrm{RR}^{b}$ & NHW RR $^{c}$ \\
& $(95 \% \mathrm{CI})$ & $(95 \% \mathrm{CI})$ & $(95 \% \mathrm{CI})$ & $(95 \% \mathrm{CI})$ \\
All other female & $0.70^{*}$ & $0.73^{*}$ & $1.17^{*}$ & $0.80^{*}$ \\
cancer sites & $(0.69-0.70)$ & $(0.72-0.73)$ & $(1.16-1.19)$ & $(0.79-0.81)$ \\
Breast & $1.10^{*}$ & $0.73^{*}$ & $2.02^{*}$ & $0.78^{*}$ \\
& $(1.08-1.11)$ & $(0.72-0.75)$ & $(1.94-2.11)$ & $(0.73-0.82)$ \\
\hline
\end{tabular}

NHW: Non-Hispanic White; NHB: Non-Hispanic Black; SE, standard error of mean; RR: rate ratio; CI: confidence interval; NOS: not otherwise specified; Rates are per 100,000 and age-adjusted to the 2000 US standard population (19 age groups - Census P25-1130) standard.

The rate ratio indicates that the rates are significantly different $(P<0.05)$.

a Younger women was defined as those aged 15-44 years.

$b$ The NHB-to-NHW ratio for younger women was the age-adjusted incidence and mortality rates for NHB women aged $15-44$ years divided by the age-adjusted incidence and mortality rates for NHW aged 15-44 years, and age-adjusted incidence and mortality rates obtained from the 20092013 NPCR (National Program of Cancer Registries) and SEER (Surveillance, Epidemiology, and End Results) registries.

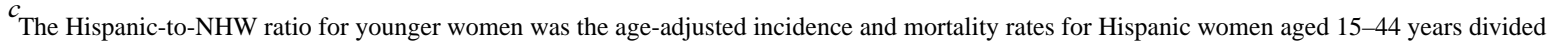
by the age-adjusted incidence and mortality rates for NHW women aged 15-44 years, and age-adjusted incidence and mortality rates obtained from the 2009-2013 NPCR and SEER registries. 